\title{
Valuing Path Dependent Options in the Variance-Gamma Model by Monte Carlo with a Gamma Bridge
}

Claudia Riveiro and Nick Webber 


\title{
Valuing Path Dependent Options in the Variance-Gamma Model by Monte Carlo with a Gamma Bridge
}

\author{
Claudia Ribeiro \\ University of Warwick \\ Warwick Business School \\ Coventry CV4 7AL \\ United Kingdom \\ Tel: +44 2476528432
}

Email: Claudia.Ribeiro@wbs.ac.uk

\author{
Nick Webber* \\ City University \\ Cass Business School \\ London EC2Y 8HB \\ United Kingdom
}

Tel: +44 2070405171

Email: Nick.Webber@city.ac.uk

September 20, 2002

\begin{abstract}
The Variance-Gamma model has analytical formulae for the values of European calls and puts. These formulae have to be computed using numerical methods. In general, option valuation may require the use of numerical methods including PDE methods, lattice methods, and Monte Carlo methods.

We investigate the use of Monte Carlo methods in the Variance-Gamma model. We demonstrate how a gamma bridge process can be constructed. Using the bridge together with stratified sampling we obtain considerable speed improvements over a plain Monte Carlo method.

The method is illustrated by pricing lookback, average rate and barrier options in the Variance-Gamma model. We find the method is up to around 400 times fast than plain Monte Carlo.
\end{abstract}

\footnotetext{
${ }^{*}$ Corresponding author. Claudia Ribeiro gratefully acknowledges the support of Fundação para a Ciência e a Tecnologia and Faculdade de Economia, Universidade do Porto. The paper has benefited from comments from Lynda McCarthy.
} 


\section{Introduction}

The variance-gamma model has been investigated by a number of authors for application to option valuation (Madan and Seneta (90) [16], Madan and Milne (91) [15], Madan, Carr and Chang (98) [14], Ané (99) [4] and Carr, Geman, Madan and Yor (01) [7]). Although analytical solutions are available for European-style options, other options require the use of numerical methods. These include Monte Carlo methods, Fourier transform (FFT) methods (Carr and Madan (99) [8]), and PDE approaches (the 'method of lines'; Albanese, Jaimungal and Rubisov (01a), (01b) [1], [2]).

This paper investigates the use of Monte Carlo methods with the variancegamma model. In particular we show how a gamma bridge may be constructed and used in conjunction with stratified sampling. We demonstrate that considerable efficiency gains are possible. If improved algorithms for sampling certain distributions become available these gains may be further improved.

The gamma bridge can be used with, augment and supplement control variate methods, importance sampling methods and other variance reduction techniques. ${ }^{1}$

The second section of this paper recaps the variance-gamma process and its application to option pricing. We review how Monte Carlo methods may be applied by exploiting the subordinated Brownian motion representation of the variance-gamma process. In the third section we show how a gamma-bridge can be constructed and applied. The fourth section presents numerical results and the fifth section concludes.

\section{The Variance-Gamma Framework}

We review the variance-gamma process and its application to option pricing. We describe a 'plain' Monte Carlo method related to the subordinated Brownian motion representation of the variance-gamma process.

\subsection{The Variance-Gamma Process}

A variance-gamma process $X_{t}$ has three parameters: $\mu \in \mathbb{R}, \sigma>0, \nu>0$. It is pure jump with Lévy density $k_{X}(x)$,

$$
k_{X}(x) \mathrm{d} x=\frac{\exp \left(\frac{\mu x}{\sigma^{2}}\right)}{\nu|x|} \exp \left(-\frac{1}{\sigma} \sqrt{\frac{2}{\nu}+\frac{\mu^{2}}{\sigma^{2}}}|x|\right) \mathrm{d} x
$$

\footnotetext{
${ }^{1}$ Indeed a suitable delta control variate for the variance-gamma model of asset returns is the delta of the option value generated by ordinary geometric Brownian motion. Since the density function of the variance-gamma process is known, importance sampling can be applied by using shifted mean and variance parameters to sample more closely the region of the distribution of interest.
} 
and characteristic function

$$
\mathbb{E}\left[\exp \left(i u X_{t}\right)\right]=\left(\frac{1}{1-i \mu \nu u+\frac{1}{2} \sigma^{2} \nu u^{2}}\right)^{\frac{ \pm}{\nu}}
$$

The density function $f_{t}^{V G}(x)$ of $X_{t}$ may be found explicitly. It is

$$
f_{t}^{V G}(x)=\frac{2 \exp \left(\frac{\mu x}{\sigma^{2}}\right)}{\nu^{\frac{t}{\nu}} \sqrt{2 \pi} \sigma \Gamma\left(\frac{t}{\nu}\right)}\left(\frac{x^{2}}{\frac{2 \sigma^{2}}{\nu}+\mu^{2}}\right)^{\frac{t}{2 \nu}-\frac{1}{4}} K_{\frac{t}{2 \nu}-\frac{1}{2}}\left(\frac{1}{\sigma^{2}} \sqrt{x^{2}\left(\frac{2 \sigma^{2}}{\nu}+\mu^{2}\right)}\right)
$$

where $K_{v}(z)$ is the modified Bessel function of the third kind,

$$
K_{v}(z)=\frac{1}{2} \int_{0}^{\infty} y^{\nu-1} \exp \left(-\frac{1}{2} z\left(y+y^{-1}\right)\right) \mathrm{d} y .
$$

$X_{t}$ can be represented as a subordinated Brownian motion, $X_{t}=w_{h(t)}$, where $w_{t}$ is Brownian motion with drift $\mu$ and variance $\sigma^{2}$ and $h(t)$ is a gamma process $h_{t} \sim \mathrm{G}\left(\frac{t}{\nu}, \nu\right) \sim \nu \mathrm{G}\left(\frac{t}{\nu}\right)$. The density $f_{t}^{h}(x)$ of $h_{t}$ conditional on $h(0)=$ 0 is

$$
f_{t}^{h}(x)=\frac{x^{\frac{t}{\nu}-1} \exp \left(-\frac{x}{v}\right)}{\nu^{\frac{t}{\nu}} \Gamma\left(\frac{t}{\nu}\right)} .
$$

$f_{t}^{V G}(x)$ can be obtained directly from this representation since

$$
f_{t}^{V G}(x)=\int_{0}^{\infty} \frac{1}{\sigma \sqrt{2 \pi g}} \exp \left(-\frac{1}{2}\left(\frac{x-\mu g}{\sigma \sqrt{g}}\right)^{2}\right) \frac{g^{\frac{t}{\nu}-1} \exp \left(-\frac{g}{v}\right)}{\nu^{\frac{1}{\nu}} \Gamma\left(\frac{t}{\nu}\right)} \mathrm{d} g .
$$

The variance gamma process can be used to model stock price returns. Let $S_{t}$ be the stock price at time $t$. In this exposition we assume that the stock pays no dividends. We take the state space $\Omega$ to be the path space of $X_{t}$ equipped with the filtration induced by the variance-gamma process. Following Madan, Carr and Chang (98) [14], Barndorff-Nielsen and Shephard (00) [5], Eberlein and Keller (95) [12], et cetera, we model a stock price process $S_{t}$ under the pricing measure ${ }^{2} F$ as

$$
S_{t}=S_{0} \exp \left(r t+X_{t}-\varpi t\right)
$$

where $X_{t}$ is a variance-gamma process, $r$ is the short rate, a constant, and the presence of the compensator $\varpi$, defined by $\mathrm{e}^{\varpi}=\mathbb{E}\left[\exp \left(X_{1}\right)\right]$, ensures that $S_{t} \mathrm{e}^{-r t}$ is a martingale under the measure associated with the accumulator account numeraire. From (2) we have

$$
\varpi=-\frac{1}{\nu} \ln \left(1-\mu \nu-\frac{1}{2} \sigma^{2} \nu\right)
$$

The $\log$ price relative $z_{t}=\ln \left(S_{t} / S_{0}\right)$ of $S_{t}$ has density $f_{t}^{z}(x)=f_{t}^{V G}\left(x^{\prime}\right)$ where $x^{\prime}=x-r t-\frac{t}{\nu} \ln \left(1-\mu \nu-\sigma^{2} \nu / 2\right)$.

\footnotetext{
${ }^{2}$ In general there is no unique martingale measure for a Lévy process. Since we focus on numerical solutions for processes of the form (7) we do not pursue this issue further.
} 


\subsection{Monte Carlo Methods for the Variance-Gamma Pro- cess}

Suppose an option has payoff $H_{T} \equiv H_{T}(\omega)$ at time $T$, where $H_{T}$ may depend on the state $\omega \in \Omega$. Under the martingale measure $F$ associated with the accumulator numeraire the option value $c_{t}$ at time $t<T$ is

$$
c_{t}=\mathbb{E}\left[H_{T} \mathrm{e}^{-r(T-t)}\right]
$$

In this section we recall how (9) can be solved using plain Monte Carlo. A standard reference for applications of Monte Carlo methods in finance is Jäckel (02) [13].

The valuation equation (9) is an integral over the state space $\Omega$,

$$
c_{t}=\mathbb{E}\left[H_{T} \mathrm{e}^{-r(T-t))}\right]=\mathrm{e}^{-r(T-t)} \int_{\Omega} H_{T}(\omega) \mathrm{d} F(\omega)
$$

The integral can be approximated by constructing a set $\left\{\widehat{\omega}^{m}\right\}_{m=1, \ldots, M}$ of discrete sample paths randomly selected under a measure $\widehat{F}$, a discrete approximation to the measure $F$. Then the approximation $\widehat{c}_{t}$ to $c_{t}$ is

$$
\widehat{c}_{t}=\mathrm{e}^{-r(T-t)} \frac{1}{M} \sum_{m=1}^{M} H_{T}\left(\widehat{\omega}^{m}\right) .
$$

Discrete sample paths for a subordinated Brownian motion, $X_{t}=w_{h(t)}$, can be constructed by first constructing discrete sample paths for the subordinator $h(t)$ and then sampling the process $w_{t}$ at times determined by the paths found for $h(t)$.

We construct discrete sample paths for $X_{t}$ over the period $[0, T]$ with $N$ time steps at times $0=t_{0}<t_{1}<\ldots<t_{N}=T$. First we construct a discrete sample path $\left\{\widehat{h}_{n}\right\}_{n=0, \ldots, N}$ for $h(t)$. Set $\widehat{h}_{0}=0$. Iteratively, $\widehat{h}_{n+1}-\widehat{h}_{n}$ is a random increment in $h$ over the interval $\Delta t_{n}=t_{n+1}-t_{n}$. For the variance-gamma process, $\Delta \widehat{h}_{n}=\widehat{h}_{n+1}-\widehat{h}_{n} \sim \mathrm{G}\left(\frac{\Delta t_{n}}{\nu}, \nu\right)$ and is easy to simulate. Given the path $\left\{\widehat{h}_{n}\right\}_{n=0, \ldots, N}$, and setting $\widehat{w}_{0}=0$, we set iteratively $\Delta \widehat{w}_{n}=\widehat{w}_{n+1}-\widehat{w}_{n} \sim$ $\mathrm{N}\left(\mu \Delta \widehat{h}_{n}, \sigma^{2} \Delta \widehat{h}_{n}\right)$. The path $\widehat{w}=\left\{\widehat{w}_{n}\right\}_{n=0, \ldots, N}$ is a discrete approximation to a sample path $\omega$ of $X_{t}$.

The plain Monte Carlo method constructs $M$ discrete sample paths $\left\{\widehat{w}^{m}\right\}_{m=1, \ldots, M}$, as above. The plain Monte Carlo estimate is then given by (11).

We may assume elsewhere that the time step $\Delta t=\frac{T}{N}$ is a constant. This assumption is for simplicity only and may be relaxed trivially. 


\section{A Gamma Bridge for the Variance-Gamma Process}

The plain Monte Carlo estimate $\widehat{c}_{t}$ converges to $c_{t}$ as $M$ and $N$ go to infinity. However, convergence to within an error bound may be very slow. ${ }^{3}$ An effective Monte Carlo method requires effective speed-ups. When simulating a Brownian motion, a Brownian bridge is often used in conjunction with stratified sampling. This technique helps to ensure that the set $\left\{\widehat{w}^{m}\right\}_{m=1, \ldots, M}$ of discrete sample paths is drawn more evenly under the measure $\widehat{F}$. In this section we review the method of stratified sampling and the use of a bridge for a stochastic process and its application to a Wiener process. We describe the construction of the gamma bridge and its application to the variance-gamma process. The algorithm, 'bridge' Monte Carlo, is presented.

\subsection{Stratified Sampling}

Initially suppose that the payoff function $H_{T}$ depends solely on the value $X_{T}$ of a state variable at time $T$, with distribution function $F_{T}^{X}(x)$ and density function $f_{T}^{X}(x)$. Then

$$
\begin{aligned}
c_{t} & =\mathrm{e}^{-r(T-t)} \int_{\Omega} H_{T}(\omega) \mathrm{d} F(\omega) \\
& =\mathrm{e}^{-r(T-t)} \int H_{T}(x) f_{t}^{X}(x) \mathrm{d} x \\
& \sim \mathrm{e}^{-r(T-t)} \frac{1}{M} \sum_{m=1}^{M} H_{T}\left(\widehat{X}^{m}\right)
\end{aligned}
$$

where $\widehat{X}^{m}$ is drawn from the distribution $F_{T}^{X}$.

A stratified sample of size $M$ from $F_{T}^{X}$ is one in which the $m$ th draw, $\widehat{X}^{m}$, is constructed to lie in the $m$ th quantile band, $\left[\frac{m-1}{M}, \frac{m}{M}\right]$, for $1 \leq m \leq M$. Given a sample $\left\{\widehat{v}^{m}\right\}_{m=1, \ldots, M}$ drawn from $\mathrm{U}[0,1]$, the set $\left\{\widehat{u}^{m}\right\}_{m=1, \ldots, M}$, where $\widehat{u}^{m}=$ $\frac{m-1+\widehat{v}^{m}}{M}$, is a stratified sample of $\mathrm{U}[0,1]$. An alternative to this Monte Carlo stratification method is to sample $\mathrm{U}[0,1]$ using a low discrepancy sequence. If the function $\left(F_{T}^{X}\right)^{-1}$ is known, and given a stratified sample $\left\{\widehat{u}^{m}\right\}_{m=1, \ldots, M}$ of the uniform distribution $\mathrm{U}[0,1]$, then the set $\left\{\left(F_{T}^{X}\right)^{-1}\left(\widehat{u}^{m}\right)\right\}_{m=1, \ldots, M}$ is a stratified sample from the distribution $F_{T}^{X}$. We use this technique, the inverse transform method, in the sequel.

An option value constructed using a stratified sample may have an actual standard deviation significantly less the size of that of a value found using plain Monte Carlo. ${ }^{4}$

\footnotetext{
${ }^{3}$ Benchmark comparisons are presented in the next section.

${ }^{4}$ This is because a stratified sample has autocorrelation. We find the actual standard deviation may be a tenth or less than the standard error.
} 
If $H_{T}$ depends on an entire sample path, as is the case for an average rate option, a set of sample paths may be found by first finding a stratified sample $\left\{\widehat{X}^{m}\right\}_{m=1 \ldots, M}$ from the terminal time, and then constructing a path $0=\widehat{X}_{0}^{m}<\widehat{X}_{1}^{m}<\ldots<\widehat{X}_{N}^{m}=\widehat{X}_{m}$ so that each $\widehat{X}_{n}^{m}$ has the correct conditional distribution. This set of paths will sample from $\widehat{F}$ more evenly than a sample without stratification. We call the path $\left\{\widehat{X}_{n}^{m}\right\}_{n=0, \ldots, N}$, constructed from $\widehat{X}_{0}^{m}$ and $\widehat{X}_{N}^{m}$, an $X$-bridge. Intermediate points $\widehat{X}_{n}^{m}$ are constructed by sampling from a bridge distribution, defined and described in the next section. This sampling may also be stratified, leading to improved sampling at the intermediate times and of the path as a whole.

\subsection{A Bridge for a Wiener Process}

Suppose that $x \sim F_{x}$ and $y \sim F_{y}$ are random variables with distributions $F_{x}$ and $F_{y}$, densities $f_{x}$ and $f_{y}$ and joint density function $f_{x, y}$. Set $z=x+y$ with density $f_{z}$. We are interested in the conditional distribution of $x \mid z$. Write $f_{x \mid z}$ for the density of $x \mid z$. Then

$$
f_{x \mid z}(x)=\frac{f_{x, y}(x, z-x)}{f_{z}(z)} .
$$

If $x$ and $y$ are independent then $f_{x \mid z}(x)=f_{x}(x) f_{y}(z-x) / f_{z}(z)$.

When $x$ and $y$ represent increments in a Markov stochastic process their densities will depend on the time increment. For instance, for a Wiener process $w_{t}$ where $x=w_{t_{j}}-w_{t_{i}}$ is a random increment between times $t_{i}$ and $t_{j}$ and $y=w_{t_{k}}-w_{t_{j}}$ is a random increment between times $t_{j}$ and $t_{k}$, then $x \sim \mathrm{N}\left(0, \sigma_{x}^{2}\right)$, $y \sim \mathrm{N}\left(0, \sigma_{y}^{2}\right), z \sim \mathrm{N}\left(0, \sigma_{z}^{2}\right)$ where $\sigma_{x}^{2}=t_{j}-t_{i}, \sigma_{y}^{2}=t_{k}-t_{j}$ and $\sigma_{z}^{2}=t_{k}-t_{i}$. In this case the density $f_{x \mid z}(x)$ is

$$
\begin{aligned}
f_{x \mid z}(x) & =\frac{1}{\sqrt{2 \pi}} \frac{\sigma_{z}}{\sigma_{x} \sigma_{y}} \exp \left(-\frac{1}{2}\left(\frac{x^{2}}{\sigma_{x}^{2}}+\frac{(z-x)^{2}}{\sigma_{y}^{2}}-\frac{z^{2}}{\sigma_{z}^{2}}\right)\right) \\
& =\frac{1}{\sqrt{2 \pi}} \frac{1}{b} \exp \left(-\frac{1}{2}\left(\frac{x-a z}{b}\right)^{2}\right)
\end{aligned}
$$

where

$$
\begin{aligned}
& a=\frac{\sigma_{x}^{2}}{\sigma_{z}^{2}}=\frac{t_{j}-t_{i}}{t_{k}-t_{i}} \\
& b=\frac{\sigma_{x} \sigma_{y}}{\sigma_{z}}=\sqrt{\frac{\left(t_{j}-t_{i}\right)\left(t_{k}-t_{j}\right)}{\left(t_{k}-t_{i}\right)}} .
\end{aligned}
$$

Hence, given $z=w_{t_{k}}-w_{t_{i}}, x=w_{t_{j}}-w_{t_{i}}$ is normally distributed, $x \sim$ 


$$
\begin{aligned}
& \mathrm{N}\left(z a, b^{2}\right) \sim \mathrm{N}\left(z \frac{t_{j}-t_{i}}{t_{k}-t_{i}}, \frac{\left(t_{j}-t_{i}\right)\left(t_{k}-t_{j}\right)}{\left(t_{k}-t_{i}\right)}\right), \text { so that } \\
& w_{t_{j}}=\frac{t_{k}-t_{j}}{t_{k}-t_{i}} w_{t_{i}}+\frac{t_{j}-t_{i}}{t_{k}-t_{i}} w_{t_{k}}+\sqrt{\frac{\left(t_{j}-t_{i}\right)\left(t_{k}-t_{j}\right)}{\left(t_{k}-t_{i}\right)}} \varepsilon_{t_{j}},
\end{aligned}
$$

for $\varepsilon_{t_{j}} \sim N(0,1)$. This is the bridge distribution for a Brownian bridge.

\subsection{A Bridge for a Gamma Process}

Consider a gamma process $g_{t}$ observed at times $t_{i}, t_{j}$ and $t_{k}$. Set $\tau_{x}=t_{j}-t_{i}$, $\tau_{y}=t_{k}-t_{j}$ and $\tau_{z}=t_{k}-t_{i}$. Let $x$ be an increment in $g_{t}$ over the period $\left[t_{i}, t_{j}\right], y$ an increment over $\left[t_{j}, t_{k}\right]$ and $z$ an increment over $\left[t_{i}, t_{k}\right]$. Then $x=$ $g_{t_{j}}-g_{t_{i}} \sim \mathrm{G}\left(\frac{\tau_{x}}{\nu}, v\right), y=g_{t_{k}}-g_{t_{j}} \sim \mathrm{G}\left(\frac{\tau_{y}}{\nu}, v\right)$ and $z=g_{t_{k}}-g_{t_{i}} \sim \mathrm{G}\left(\frac{\tau_{z}}{\nu}, v\right)$. The conditional density $f_{x \mid z}(x)$ is

$$
\begin{aligned}
f_{x \mid z}(x) & =\frac{x^{\frac{\tau_{x}}{\nu}}-1 \exp \left(-\frac{x}{v}\right)}{\nu^{\frac{\tau_{x}}{\nu}} \Gamma\left(\frac{\tau_{x}}{\nu}\right)} \frac{(z-x)^{\frac{\tau_{y}}{\nu}-1} \exp \left(-\frac{z-x}{v}\right)}{\nu^{\frac{\tau_{y}}{\nu}} \Gamma\left(\frac{\tau_{y}}{\nu}\right)} \frac{\nu^{\frac{\tau_{z}}{\nu}} \Gamma\left(\frac{\tau_{z}}{\nu}\right)}{z^{\frac{\tau_{z}}{\nu}-1} \exp \left(-\frac{z}{v}\right)} \\
& =\frac{1}{2} \frac{\Gamma\left(\frac{\tau_{x}}{\nu}+\frac{\tau_{y}}{\nu}\right)}{\Gamma\left(\frac{\tau_{x}}{\nu}\right) \Gamma\left(\frac{\tau_{y}}{\nu}\right)}\left(\frac{x}{z}\right)^{\frac{\tau_{x}}{\nu}-1}\left(1-\frac{x}{z}\right)^{\frac{\tau_{y}}{\nu}-1} .
\end{aligned}
$$

Change variable to $p=\frac{x}{z}$, then $p=\frac{x}{z} \sim \mathrm{B}\left(\frac{\tau_{x}}{\nu}, \frac{\tau_{y}}{\nu}\right)$ has a beta distribution with parameters $\frac{\tau_{x}}{\nu}$ and $\frac{\tau_{y}}{\nu}$. Given $g_{t_{k}}$ and $g_{t_{i}}, \frac{x}{z}=\frac{g_{t_{j}}-g_{t_{i}}}{g_{t_{k}}-g_{t_{i}}}$ has a beta distribution so

$$
g_{t_{j}}=g_{t_{i}}+\beta_{t_{j}}\left(g_{t_{k}}-g_{t_{i}}\right)
$$

for $\beta_{t_{j}} \sim \mathrm{B}\left(\frac{t_{j}-t_{i}}{\nu}, \frac{t_{k}-t_{j}}{\nu}\right)$. This is the bridge distribution for a gamma process.

This result is intuitive. A beta variate takes values in the interval $[0,1]$. The gamma process is an increasing process. Given the increment $z$ over the pe$\operatorname{riod}\left[t_{i}, t_{k}\right]$, the beta distribution samples from the proportion of this increment achieved by time $t_{j}$.

\subsection{Application of the Gamma Bridge}

To apply bridge Monte Carlo we use the subordinator representation of the variance-gamma process and stratify it at the terminal time. We construct a bridge to the points we have constructed. The bridge may itself be stratified at intermediate times.

Suppose $X_{t}=w_{h(t)}$ for a Brownian motion $w_{t} \sim \mathrm{N}\left(\mu t, \sigma^{2} t\right)$ and subordinator $h_{t} \sim \mathrm{G}\left(\frac{t}{\nu}, \nu\right)$. Time is discretised into $N$ time steps, $0=t_{0}, t_{1}, \ldots, t_{N}=T$, up to the terminal time $T$. For a sample of size $M$ we construct:

1. A stratified sample $\left(\widehat{u}_{i}, \widehat{v}_{i}\right), i=1, \ldots, M$, from the unit hypercube of dimension 2. 
2. A stratified sample $\widehat{h}_{N}^{i}, i=1, \ldots, M$, of the subordinator at time $t_{N}=T$, using the variates $\widehat{u}_{i}, i=1, \ldots, M$. Set $\widehat{h}_{N}^{i}=G_{T}^{-1}\left(\widehat{u}_{i}\right)$, where $G$ is the distribution function for $h_{T}$.

3. An $h$-bridge $\widehat{h}^{i}=\left(\widehat{h}_{0}^{i}, \ldots, \widehat{h}_{N}^{i}\right)$, for $i=1, \ldots, M$. We discuss below how the $h$-bridge may be further stratified at intermediate times.

4. For each $i=1, \ldots, M$ a sample point $\widehat{w}_{N}^{i} \sim \mathrm{N}\left(\mu \widehat{h}_{N}^{i}, \sigma^{2} \widehat{h}_{N}^{i}\right), \widehat{w}_{N}^{i}=$ $N_{\mu \hat{h}_{N}^{i}, \sigma^{2} \widehat{h}_{N}^{i}}^{-1}\left(\widehat{v}_{i}\right)$, where $N_{\mu, \sigma^{2}}$ is the normal distribution function with mean $\mu$ and variance $\sigma^{2}$.

5. A $w$-bridge $\widehat{w}^{i}=\left(\widehat{w}_{0}^{i}, \ldots, \widehat{w}_{N}^{i}\right)$, at times $\widehat{h}_{0}^{i}, \ldots, \widehat{h}_{N}^{i}, i=1, \ldots, M$. This is an $X$-bridge for times $t_{0}, \ldots, t_{N}$. The $w$-bridge may be stratified further at intermediate times.

The set of paths $\widehat{w}^{i}, i=1, \ldots, M$, is a stratified bridge sample from the path space of the Lévy process $X_{t}$.

Note that we stratify both $h_{t}$ and $w_{h(t)}$ at the terminal time. We found experimentally that full speed-ups were not achieved unless both the subordinator and the Brownian motion were stratified. In the sequel we always stratify both $h_{t}$ and $w_{h(t)}$.

\subsection{Stratifying the Variance-Gamma Bridge}

For $t_{i}<t_{k}$, given $g_{t_{i}}$ and $g_{t_{k}}$, the value $g_{t_{j}}$ of a gamma process at an intermediate time $t_{j}$ is generated as $g_{t_{j}}=g_{t_{i}}+\beta_{t_{j}}\left(g_{t_{k}}-g_{t_{i}}\right)$ for $\beta_{t_{j}} \sim \mathrm{B}\left(\frac{t_{j}-t_{i}}{\nu}, \frac{t_{k}-t_{j}}{\nu}\right)$. A stratified sample for $\beta_{t_{j}}$ yields a stratified sample for $g_{t_{j}}$. We obtain such a sample by inverse transform from a stratified sample $\widehat{u}_{n}, n=1, \ldots, M$ of the unit interval. Set $\beta_{t_{j}}^{n}=B_{\frac{t_{j}-t_{i}}{\nu}}^{-1} \frac{t_{k}-t_{j}}{\nu}\left(\widehat{u}_{n}\right)$, where $B_{\alpha, \beta}^{-1}$ is the inverse of the cumulative distribution function $B(\alpha, \beta)$.

If the gamma process is stratified at time $t_{j}$ then we also stratify $w$ at time $h_{t_{j}}$.

We present in the next section comparisons of bridge Monte Carlo when stratified at different numbers of times. To stratify at $K$ times, where $K=2^{P}$ and $N=Q K$ for integer $P$ and $Q$, we first compute a stratified sample of points $h_{N}^{i}$ and $w_{N}^{i}, i=1, \ldots, M$, at time $t_{N}$. We then stratify successively at times $\frac{1}{2} t_{N}, \frac{3}{4} t_{N}, \frac{1}{4} t_{N}, \frac{7}{8} t_{N}, \frac{5}{8} t_{N}, \frac{3}{8} t_{N}, \frac{1}{8} t_{N}$, and so on, until all times $\frac{k}{K} t_{N}, k=$ $1, \ldots, K$ have been stratified. The remaining intermediate points (when $Q>1$ ) are filled in using ordinary random draws from beta and normal distributions. We stratify by constructing a stratified sample from a $2 K$-dimensional unit hypercube. For $K \leq 2$ it may be plausible to use a Monte Carlo stratified sample. In the numerical section, except where noted, we use low discrepancy sampling for all $K \geq 1$. 


\section{Numerical Results}

We first benchmark the bridge Monte Carlo method against European call option values. Then we use the bridge to value average rate, lookback and barrier options and compare the results to those found with plain Monte Carlo. The performance of the bridge under various degrees of stratification is investigated.

All the path dependent instruments mature in one year. We investigate reset frequencies from quarterly to approximately daily. We find that the bridge method benchmarks very accurately, achieving efficiency gains of a factor of 50 for European calls with one year to maturity. For path dependent options with daily resets and 16 stratification times we achieve gains of a factor of 130 for lookback options and 380 for average rate options. Gains are also found for barrier options but these may be significantly less. For our examples one stratification (at the terminal time) is ten to twenty times faster than plain Monte Carlo, but further stratification does not always bring further efficiency gains.

\subsection{Algorithm Issues}

We require algorithms for generating uniform, normal, gamma and beta random variates.

Uniform variates are generated using a VBA version of ran2 from Numerical Recipes (92) [18]. All normal variates were generated by inverse transform. $N^{-1}$, the inverse of the normal distribution function, is computed using Applied Statistics Algorithm 111 [3] downloadable from lib.stat.cmu.edu/apstat/111.

To generate gamma variates directly we use the Best (83) and Best (78) algorithms as described in Devroye (86) [10]. To compute the inverse of the gamma distribution function, $G^{-1}$, to use with the inverse transform method for stratified sampling, we use the algorithm of DiDonato and Morris (87) [11], downloadable from www.netlib.org/toms/654. It uses an iterative method to find solutions of $G\left(g_{t_{N}}^{n}\right)-\widehat{u}_{n}=0$.

Beta variates from the distribution $\mathrm{B}(\alpha, \beta)$ are generated directly by Cheng's method if $\min (\alpha, \beta)<1$, Johnk's method if $\max (\alpha, \beta)<1$, by Atkinson and Whittaker's method if $\min (\alpha, \beta)<1<\max (\alpha, \beta)$, and by ratio of gammas otherwise. $^{5}$ For stratified sampling, the inverse of the beta distribution function, $B^{-1}$, is computed using an algorithm due to Moshier (00) [17]. This algorithm uses an iterative method to solve for $B_{\frac{\tau_{x}}{\nu}, \frac{\tau_{y}}{\nu}}\left(\beta_{t_{j}}^{n}\right)-\widehat{u}_{n}=0$. We shall see that this particular procedure is relatively slow compared, for instance, to computing $N^{-1}$. Should faster algorithms emerge to compute $B_{\frac{\tau_{x}}{\nu}, \frac{\tau_{y}}{y}}^{-1}$ (or indeed $G_{\frac{\tau_{y}}{y}, v}^{-1}$ ) then the efficiency gains to the algorithm would be even greater than those we find below.

For low discrepancy sampling we use a Sobol' sequence based on Bratley and Bennett (88) [6]. Code is downloadable from www.netlib.org/toms/659. The

${ }^{5}$ See [9] and [10]. Johnk's method sometimes fails when both $\alpha$ and $\beta$ are small. In these cases we revert to the ratio of gammas method. 


\begin{tabular}{|l|l|c|c|c|}
\hline \multirow{2}{*}{ Maturity } & Explicit & \multicolumn{3}{|c|}{ Monte Carlo } \\
\cline { 3 - 5 } 0.25 & 3.4742 & $\begin{array}{c}3.4548 \\
(0.035)\end{array}$ & $\begin{array}{c}3.4748 \\
(0.003)\end{array}$ & 25 \\
\hline 0.5 & 6.2406 & $\begin{array}{r}6.2414 \\
(0.056)\end{array}$ & $\begin{array}{c}6.2401 \\
(0.004)\end{array}$ & 35 \\
\hline 0.75 & 8.6909 & $\begin{array}{c}8.6928 \\
(0.077)\end{array}$ & $\begin{array}{c}8.6955 \\
(0.005)\end{array}$ & 43 \\
\hline 1 & 10.9815 & $\begin{array}{c}10.9381 \\
(0.099)\end{array}$ & $\begin{array}{c}10.9721 \\
(0.006)\end{array}$ & 50 \\
\hline Time: & - & 0.3 & 1.4 & \\
\hline
\end{tabular}

Table 1: Comparison of Plain and Stratified Monte Carlo: Calls, one time step.

code generates low discrepancy samples from a unit hypercube of dimension at most 39. Since bridge Monte Carlo uses two low discrepancy coordinates at each stratified time, we are constrained to have at most 18 stratification times.

\subsection{Benchmarking to European calls}

We value European calls and compare values obtained from an analytic formula ("Explicit") against Monte Carlo values. ${ }^{6}$ Table 1 compares plain Monte Carlo with stratified Monte Carlo, taking 10,000 samples directly from the terminal distribution for each maturity $(M=10,000, N=1)$. Explicit values are computed using the analytical formula of Madan, Carr and Chan (98) [14]. Standard deviations are shown in brackets. ${ }^{7,8}$ Tables 1 and 2 use 'ordinary' Monte Carlo stratification instead of low discrepancy stratification.

For each option in table 1 plain Monte Carlo took about 26 seconds for a hundred replications. Stratified Monte Carlo took about 144 seconds.

The final column of table 1 gives the efficiency gain of the stratified Monte Carlo method over plain Monte Carlo. Suppose for some option plain Monte Carlo gives a standard deviation of $\sigma_{P}$ in time $t_{P}$ and an alternative Monte Carlo method gives $\sigma_{A}$ and $t_{A}$. The efficiency gain $E_{A P}$ of the alternative method to plain Monte Carlo is

$$
E_{A P}=\frac{\sigma_{P}^{2} t_{P}}{\sigma_{A}^{2} t_{A}} .
$$

\footnotetext{
${ }^{6}$ Call values are for options on an asset with initial value $S_{0}=100$, exercise price $X=101$ and riskless rate $r=\mathbf{0 . 1}$. Parameters of the variance-gamma process are $\mu=-\mathbf{0 . 1 4 3 6}$, $\sigma=0.12136, \nu=0.3$ (based upon Madan, Carr and Chan (98) [14]). Maturities, in years, range up to 1 year.

${ }^{7}$ For plain Monte Carlo the standard deviation is approximately equal to the standard error. For bridge Monte Carlo, in all the tables of this section, the true standard deviation is found from a hundred replications of the Monte Carlo procedure.

${ }^{8}$ All programmes were written in Visual Basic 6.0 and were run on an $800 \mathrm{Mhz}$ PC.
} 


\begin{tabular}{|c|c|c|c|c|c|c|}
\hline \multirow[b]{2}{*}{ Maturity } & \multirow[b]{2}{*}{ Explicit } & \multicolumn{5}{|c|}{ Stratified Monte Carlo: Number of sample paths, $M$} \\
\hline & & 10000 & 1600 & 900 & 400 & 225 \\
\hline 0.25 & 3.4742 & $\begin{array}{c}3.475 \\
(0.0028)\end{array}$ & $\begin{array}{c}3.476 \\
(0.011)\end{array}$ & $\begin{array}{c}3.494 \\
(0.018)\end{array}$ & $\begin{array}{c}3.436 \\
(0.037)\end{array}$ & $\begin{array}{c}3.505 \\
(0.052)\end{array}$ \\
\hline 0.5 & 6.2406 & $\begin{array}{c}6.240 \\
(0.004)\end{array}$ & $\begin{array}{c}6.242 \\
(0.017)\end{array}$ & $\begin{array}{c}6.285 \\
(0.026)\end{array}$ & $\begin{array}{c}6.268 \\
(0.050)\end{array}$ & $\begin{array}{c}6.301 \\
(0.068)\end{array}$ \\
\hline 0.75 & 8.6909 & $\begin{array}{c}8.696 \\
(0.005)\end{array}$ & $\begin{array}{c}8.717 \\
(0.020)\end{array}$ & $\begin{array}{c}8.660 \\
(0.032)\end{array}$ & $\begin{array}{c}8.666 \\
(0.066)\end{array}$ & $\begin{array}{c}8.667 \\
(0.093)\end{array}$ \\
\hline 1 & 10.9815 & $\begin{array}{c}10.972 \\
(0.006)\end{array}$ & $\begin{array}{l}10.977 \\
(0.024)\end{array}$ & $\begin{array}{l}10.925 \\
(0.032)\end{array}$ & $\begin{array}{l}11.097 \\
(0.066)\end{array}$ & $\begin{array}{l}11.036 \\
(0.126)\end{array}$ \\
\hline Time: & - & 1.4 & 0.24 & 0.13 & 0.06 & 0.04 \\
\hline
\end{tabular}

Table 2: Stratified Monte Carlo: Standard deviation against sample paths: Calls, one time step

Under the assumption that standard deviation scales inversely with the square root of the number of sample paths $M$, and that time taken is proportional to $M$, then $E_{A P}$ is the multiple of the time the plain method takes to achieve a particular standard deviation compared to the alternative method.

We note efficiency gains of 50 for one year maturity options. The efficiency appears to be increasing with increasing maturity.

Table 2 compares stratified Monte Carlo with different numbers of sample paths. The $M=10,000$ column is repeated from table 1 . In each case the gamma and the normal variates have an equal degree of stratification. For instance, for $M=10,000$, there are a hundred buckets in each dimension of the stratified 2-dimensional unit hypercube. With only 400 sample paths the standard deviation of the stratified Monte Carlo method is significantly less than that of the plain Monte Carlo in table 1 and takes less than a quarter of the time. ${ }^{9}$

Now we investigate how using bridge Monte Carlo can increase the efficiency of valuing options of different maturities simultaneously. We evolve the variancegamma process out to 1 year in four time steps of 0.25 years each, and value simultaneously the benchmark option maturing at the conclusion of each time step.

Table 3 shows benchmarked call values computed with plain Monte Carlo and with bridge Monte Carlo stratified at (i) step 4, the terminal time, (ii) steps 2 and 4 , (iii) steps $1,2,3$ and 4 , so that $K=1,2$ and 4 for the three cases. In this table $M=100,000$ for each option. Stratification is by low discrepancy sampling. At the stratification times, stratification is entirely deterministic so no standard deviation can be reported. Table 4 displays the efficiency gains achieved by bridge Monte Carlo over plain Monte Carlo.

We see that stratification gives very significant efficiency gains for options of all maturities. Additional stratification may give greater gains for options of

\footnotetext{
${ }^{9}$ Efficiency gains decrease as $M$ decreases because of fixed set-up times in the implementation of the Monte Carlo algorithm.
} 


\begin{tabular}{|l|l|c|c|c|c|}
\hline \multirow{2}{*}{ Maturity } & \multirow{2}{*}{ Explicit } & \multirow{3}{*}{ Plain MC } & \multicolumn{3}{|c|}{ Bridge MC: Stratified at steps: } \\
\cline { 4 - 6 } 0.25 & \multirow{2}{*}{3.4742} & $\begin{array}{c}3.4756 \\
(0.034)\end{array}$ & $\begin{array}{c}3.4879 \\
(0.011)\end{array}$ & $\begin{array}{c}3.4858 \\
(0.009)\end{array}$ & $\begin{array}{c}3.4738 \\
(-)\end{array}$ \\
\hline \multirow{2}{*}{0.5} & \multirow{2}{*}{6.2406} & $\begin{array}{c}6.1712 \\
(0.061)\end{array}$ & $\begin{array}{c}6.2440 \\
(0.015)\end{array}$ & $\begin{array}{c}6.2409 \\
(-)\end{array}$ & $\begin{array}{c}6.2409 \\
(-)\end{array}$ \\
\hline \multirow{2}{*}{0.75} & \multirow{2}{*}{8.6909} & $\begin{array}{c}8.6156 \\
(0.088)\end{array}$ & $\begin{array}{c}8.6811 \\
(0.026)\end{array}$ & $\begin{array}{c}8.6860 \\
(0.011)\end{array}$ & $\begin{array}{c}8.6907 \\
(-)\end{array}$ \\
\hline \multirow{2}{*}{1} & \multirow{2}{*}{10.9815} & $\begin{array}{c}10.8766 \\
(0.10)\end{array}$ & $\begin{array}{c}10.9813 \\
(-)\end{array}$ & $\begin{array}{c}10.9813 \\
(-)\end{array}$ & $\begin{array}{c}10.9813 \\
(-)\end{array}$ \\
\hline Time: & - & 0.93 & 0.25 & 0.40 & 1.3 \\
\hline
\end{tabular}

Table 3: Comparison of Plain and Stratified Monte Carlo: Calls, one to four time steps.

\begin{tabular}{|l|c|c|}
\hline & \multicolumn{2}{|c|}{ Efficiency Gains } \\
\hline Maturity & $K=1$ & $K=2$ \\
\hline 0.25 & 35 & 33 \\
\hline 0.5 & 61 & - \\
\hline 0.75 & 42 & 147 \\
\hline 1 & - & - \\
\hline
\end{tabular}

Table 4: Bridge Monte Carlo: Efficiency Gains over Plain Monte Carlo.

longer maturities.

As a further example we compute Black-Scholes implied volatilities for options maturing at times $\frac{n}{N}$ for $n=1, \ldots, N, N=64$. Figure 1 compares BlackScholes implied volatilities of variance-gamma calls for bridge Monte Carlo stratified only at the terminal time against stratification every 4 time steps. The sample size is $M=1,000$. Option prices for each maturity are computed together. With 16 stratification times (and one replication) the programme takes 9.2 seconds to run. When stratifying only at the terminal time the programme takes 2.0 seconds. Pricing is improved with greater stratification, even taking the increased run time into account, particularly at longer maturity times.

We conclude that bridge Monte Carlo benchmarks well to European calls, achieving superior accuracy to plain Monte Carlo. We now value path dependent options in the variance-gamma framework.

\subsection{Valuing path dependent options}

We value one year average rate, lookback and barrier options with various numbers of reset times up to final maturity, comparing the results to plain Monte Carlo. We use bridge Monte Carlo with various numbers of stratification times.

We report results for plain Monte Carlo with $M=1,000,000$ sample paths. The results for bridge Monte Carlo are for $M=10,000$. Actual standard 


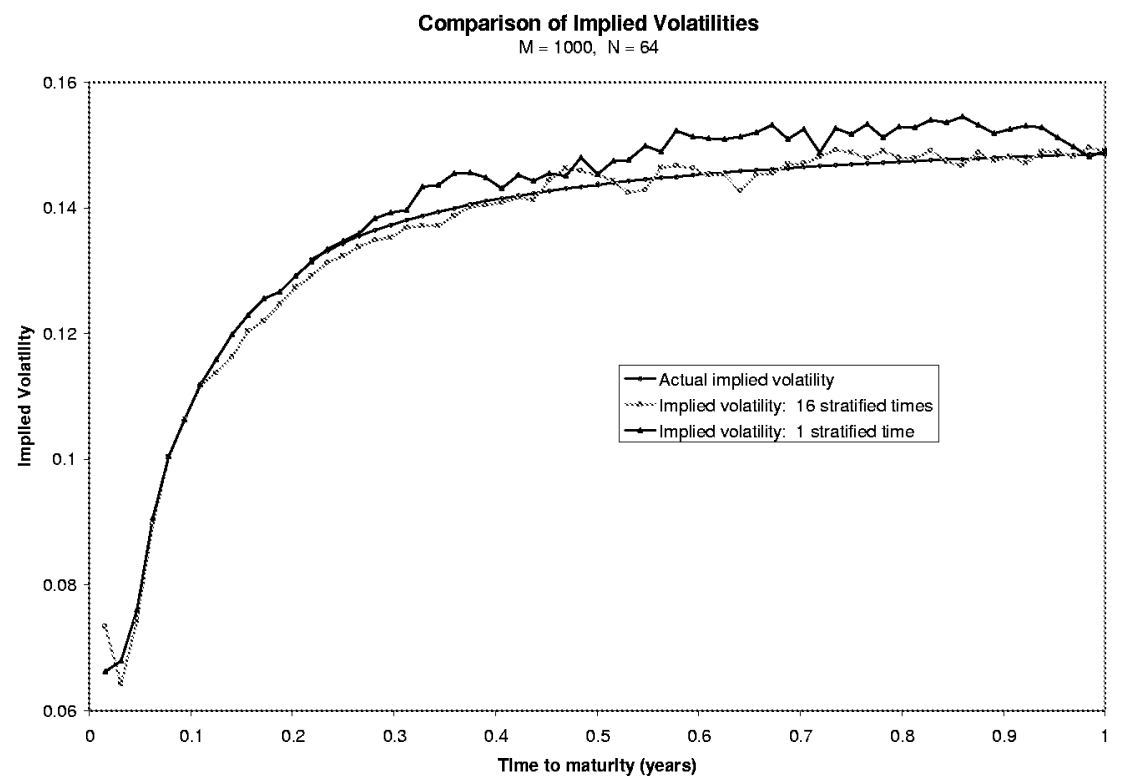

Figure 1: Comparison of implied volatilities: 1 and 16 stratification times

deviations, based on 100 replications of the full Monte Carlo procedure, are shown in round brackets. Times in seconds for a single replication are shown in square brackets. For the options investigated, the standard error and actual standard deviation of plain Monte Carlo are very similar, so only the standard error is reported.

Each option is priced under varying numbers of reset times per year, from 4 to 256 , corresponding to quarterly up to approximately daily reset frequencies. The number of times steps is equal to the number of reset times. With $N$ reset times, resets are at times $\frac{1}{N}, \frac{2}{N}, \ldots, 1$. Bridge Monte Carlo is implemented with from 1 to 16 stratification times. With $K$ stratification times, stratifications are at times $\frac{1}{K}, \frac{2}{K}, \ldots, 1$. When the number of stratification times equals the number of reset times, the method is fully low discrepancy and non-stochastic. Results in this case are based on a single replication and no standard deviation is reported. For options with 4, 8 and 16 resets we 'benchmark' by pricing using fully low discrepancy sampling with $M=1,000,000$ sample paths. We note that convergence in $M$ for fully low discrepancy methods is not uniform.

In the tables, the $K=0$ row reports the plain Monte Carlo results.

\subsubsection{Average rate options}

Table 5 shows results for average rate call options. The payoff at time one is $H_{T}=\max (A-X, 0)$ where $A$ is the arithmetic average of the asset value at 
each reset time and $X=101$ is the exercise price.

We see that the standard deviation decreases significantly with each additional level of stratification. Computation times also increase. Doubling the number of stratification times roughly halves the standard deviation but only approximately doubles the computation time. This means that each additional level of stratification is approximately doubling the efficiency gain. These are shown in Table 6.

Efficiency gains are most pronounced for options with greater numbers of reset times, but even the quarterly reset option with two stratification times is 7 times faster than plain Monte Carlo. For the daily reset case $(N=256)$ using 16 stratification times we achieve an efficiency gain of a factor of 383 over plain Monte Carlo. We have no reason to suppose that efficiency gains would not continue to increase with the introduction of further stratification times.

\subsubsection{Lookback options}

Table 7 shows results for lookback call options. The payoff at time one is $H_{T}=\max \left(S_{T}-M, 0\right)$ where $M$ is the minimum of the asset values at each reset time. Table 8 gives efficiency gains for these options.

For lookbacks we see that increasing the number of stratification times brings increasing efficiency gains, although less so than for the average rate option. The gains are most pronounced for the daily reset lookback with an efficiency gain of 129 with 16 stratification times. Efficiency gains are greater for options with fewer reset times, compared to average rate options.

\subsubsection{Barrier options}

We report results for pricing of up-and-in call options. Pricing for down-andout barrier options was also investigated but as we found similar results to the up-and-in case, these are not reported.

'Out'-type barrier options may be given a zero payoff immediately that an asset value is generated that has hit the barrier, and no further asset values along that sample path need be generated. An analogous speed-up is possible for 'In'-type barrier options. Once the barrier is hit the bridge Monte Carlo method requires no further asset values to be generated since the bridge has already generated the terminal asset value. The plain Monte Carlo method requires the generation of one further asset value, for the terminal time.

Tables 9 shows results for an up-and-in call option. The payoff at time one is $H_{T}=\max \left(S_{T}-X, 0\right)$ where $X$ is the exercise price, and where the payoff is conditional on the asset value exceeding the barrier level $B$ on at least one reset time. The table gives results for $B=120$. Table 10 gives efficiency gains for these options. We see that a single stratification, at the terminal time, gives efficiency gains from around 12 up to 23 for daily reset options. Additional stratification does not necessarily increase the efficiency, although there are gains for the daily reset option. On the whole, efficiencies do not decrease by much, implying that there is likely to be little loss from additional stratification. 
Investigation showed that efficiency gains are greater when the barrier level $B$ is further away from the initial asset value $S_{0}$. When $B$ is close to $S_{0}$ the gains are considerably less. For down-and-out options with $B=100$, efficiency gains of around 2 were obtained for one level of stratification, at the terminal time. However, further stratifications do not lead to further gains. On the contrary, for all except the option with 256 reset times, the efficiency gains diminish.

We attribute the pricing behaviour of barrier options to two factors. The first is that the values of barrier options depend on the path of asset values only through the hitting, or otherwise, of a barrier level; payoffs are not computed directly from intermediate asset values. In this sense barrier options are 'less' path dependent than average rate or lookback options whose payoffs depend directly upon intermediate asset values and whose valuation benefits from a more sophisticated sampling at intermediate times. The second factor is the efficiency of the algorithms used to compute the inverse of the beta distribution function. In fact, from table 5 , for instance, we can estimate that (for $M=$ 10,000 and with 100 replications) a 'plain' unstratified gamma bridge step takes about 0.2 seconds, the gamma stratification step at the terminal time takes about 0.6 seconds and a beta stratification step takes about 5 seconds. Were a beta stratification step to be as fast as the gamma stratification step a daily reset average rate option would have an efficiency gain of over 800 .

\section{Conclusions}

We have shown how a gamma bridge may be used in conjunction with stratified sampling in the variance-gamma model to give much improved Monte Carlo estimates of option values, both for benchmark calls and for various path dependent options. We find efficiency gains of a factor of around 380 for average rate options and 130 for lookback options. There are also gains for barrier options which are significant for a stratification at the terminal time.

The use of the bridge Monte Carlo technique should be considered whenever (i) Monte Carlo is used to value path dependent options or (ii) a single Monte Carlo run is used to price options of different maturities. From the example of barrier options it appears that the greater the effective degree of path dependence, the greater are the efficiency gains due to the use of bridge Monte Carlo.

Bridge Monte Carlo may be used to maximum effect if an efficient algorithm is available to compute the inverse of the bridge distribution function. Very good algorithms exist to compute the inverse of the normal distribution. Further efficiency gains would be possible for the gamma bridge if improved algorithms for the computation of the functions $G^{-1}$ and particularly for $B^{-1}$ were available. We would then expect to see that stratifications at intermediate times for barrier options with asset values close to the barrier level could lead to much greater efficiency gains.

In principle the bridge Monte Carlo method is widely applicable, but its ease of application depends upon the nature of the conditional distribution function 
at intermediate times, and on the efficiency of available algorithms to compute the inverse of that distribution function.

For the variance-gamma process the use of the gamma-bridge is strongly recommended for appropriate applications. 


\section{References}

[1] C. Albanese, S. Jaimungal, and D. Rubisov. Jumping in Line. Risk Magazine, $\operatorname{LV}(5): 2259-2284,2001$.

[2] C. Albanese, S. Jaimungal, and D. Rubisov. The Model of Lines for Option Pricing with Jumps. 2001. Mimeo. http://www.mathpoint.com/research.htm.

[3] Algorithm 111. Inverse of the Normal Distribution Function. Applied Statistics, 26:118-121, 1977.

[4] T. Ané. Pricing and hedging S\&P 500 index options with hermite polynomial approximation: Empirical tests of Madan and Milne's model. 1999. Mimeo.

[5] O. E. Barndroff-Nielsen and N. Shephard. Modelling by Lévy Processes for Financial Econometrics. pages 1-38, 2000. Mimeo. University of Aarhus.

[6] P Bratley and L.F Bennett. Algorithm 659 Implementing Sobol's Quasirandom Sequence Generator. Working Paper, University of Montreal, pages $88-100,88$.

[7] P. Carr, H. Geman, D. B. Madan, and M. Yor. Stochastic Volatility for Lévy Processes. April 2001.

[8] P. Carr and D. B. Madan. Option Valuation Using the Fast Fourier Transform. March 1999. Mimeo.

[9] J. Dagpunar. Principles of Random Variate Generation. Clarendon Press, 1988.

[10] L. Devroye. Non-Uniform Variate Generation. Springer-Verlang, New York, 1986.

[11] A.R. DiDonato and A.H. Morris. Incomplete gamma function ratios and their inverse. ACM TOMS, 13:318-319, 1987.

[12] E. Eberlein and U. Keller. Hyperbolic Distributions in Finance. 1995. Mimeo.

[13] P. Jackel. Monte carlo methods in finance. Wiley Finance, 2002.

[14] D. B. Madan, P. P. Carr, and E. C. Chang. The Variance Gamma Process and Option Pricing. European Finance Review, 2:79-105, 1998.

[15] D. B. Madan and F. Milne. Option Pricing with VG Martingale Components. Mathematical Finance, pages 39-55, 1(1991).

[16] D. B. Madan and E. Seneta. The Variance Gamma (V.G.) Model for Share Market Returns. Journal of Business, pages 511-524, 1990. 
[17] S.L. Moshier. Inverse of the incomplete beta integral. Cephes Math Library, 2000.

[18] W. Press, S. Teukolsky, W. Vetterling, and B. Flannery. Numerical Recipes in C. Cambridge University Press, 92. 


\begin{tabular}{|c|c|c|c|c|c|c|}
\hline \multicolumn{7}{|c|}{ Average rate call options: Times and standard deviations. } \\
\hline K & $\mathbf{4}$ resets & $\mathbf{8}$ resets & 16 resets & 32 resets & $\mathbf{6 4}$ resets & 256 resets \\
\hline \multirow{3}{*}{0} & 6.7720 & 6.0666 & 5.7274 & 5.5497 & 5.4625 & 5.4075 \\
& $(0.0064)$ & $(0.0058)$ & $(0.0055)$ & $(0.0053)$ & $(0.0052)$ & $(0.0052)$ \\
& {$[85.6]$} & {$[175.0]$} & {$[335.4]$} & {$[647.5]$} & {$[1277]$} & {$[5034]$} \\
\hline \multirow{4}{*}{1} & 6.7993 & 6.0290 & 5.7234 & 5.5242 & 5.4830 & 5.3874 \\
& $(0.029)$ & $(0.025)$ & $(0.023)$ & $(0.025)$ & $(0.024)$ & $(0.024)$ \\
& {$[2.4]$} & {$[3.5]$} & {$[5.5]$} & {$[9.3]$} & {$[17.0]$} & {$[52.2]$} \\
\hline \multirow{3}{*}{2} & 6.7635 & 6.0741 & 5.7187 & 5.5208 & 5.4761 & 5.376 \\
& $(0.011)$ & $(0.012)$ & $(0.014)$ & $(0.014)$ & $(0.013)$ & $(0.013)$ \\
& {$[4.0]$} & {$[5.0]$} & {$[6.9]$} & {$[10.8]$} & {$[18.5]$} & {$[54.9]$} \\
\hline \multirow{4}{*}{4} & 6.7594 & 6.0656 & 5.7149 & 5.5510 & 5.4765 & 5.3934 \\
& $(-)$ & $(0.0065)$ & $(0.0067)$ & $(0.0072)$ & $(0.0063)$ & $(0.0064)$ \\
& {$[13.0]$} & {$[14.0]$} & {$[16.0]$} & {$[19.9]$} & {$[27.6]$} & {$[62.9]$} \\
\hline \multirow{4}{*}{8} & - & 6.0711 & 5.7283 & 5.5465 & 5.4667 & 5.3997 \\
& $-(-)$ & $(0.0029)$ & $(0.0033)$ & $(0.0035)$ & $(0.0035)$ \\
& & {$[33.8]$} & {$[35.8]$} & {$[39.6]$} & {$[47.4]$} & {$[83.0]$} \\
\hline \multirow{2}{*}{16} & - & - & 5.7245 & 5.5527 & 5.4627 & 5.4008 \\
& & - & $(-)$ & $(0.0014)$ & $(0.0017)$ & $(0.0017)$ \\
& & & {$[77.3]$} & {$[80.7]$} & {$[88.1]$} & {$[123.1]$} \\
\hline \multirow{2}{*}{ Bench- } & 6.7626 & 6.0702 & 5.7250 & & & \\
mark & $(-)$ & $(-)$ & $(-)$ & - & - & - \\
& {$[1297]$} & {$[3370]$} & {$[7638]$} & & & \\
\hline
\end{tabular}

Table 5: Average Rate Call Options: Comparison of Plain and Bridge Monte Carlo

\begin{tabular}{|c|c|c|c|c|c|c|}
\hline \multicolumn{7}{|c|}{ Average rate call options: Efficiency gains. } \\
\hline K & $\mathbf{4}$ resets & $\mathbf{8}$ resets & $\mathbf{1 6}$ resets & 32 resets & 64 resets & 256 resets \\
\hline 1 & 1.7 & 2.7 & 3.5 & 3.1 & 2.4 & 4.5 \\
\hline 2 & 7.2 & 8.2 & 7.5 & 8.6 & 11 & 15 \\
\hline 4 & - & 10 & 14 & 18 & 32 & 53 \\
\hline 8 & - & - & 34 & 42 & 60 & 134 \\
\hline 16 & - & - & - & 115 & 136 & 383 \\
\hline
\end{tabular}

Table 6: Average Rate Call Options: Efficiency Gains for Bridge Monte Carlo over Plain Monte Carlo 


\begin{tabular}{|c|c|c|c|c|c|c|}
\hline \multicolumn{7}{|c|}{ Lookback call options: Times and standard deviations. } \\
\hline $\mathbf{K}$ & 4 resets & 8 resets & 16 resets & 32 resets & 64 resets & 256 resets \\
\hline 0 & $\begin{array}{c}13.2481 \\
(0.0097) \\
{[87.7]}\end{array}$ & $\begin{array}{c}13.8007 \\
(0.0095) \\
{[175.7]}\end{array}$ & $\begin{array}{c}14.1696 \\
(0.0094) \\
{[337.3]}\end{array}$ & $\begin{array}{c}14.3828 \\
(0.0093) \\
{[651.7]}\end{array}$ & $\begin{array}{c}14.4969 \\
(0.0093) \\
{[1276]}\end{array}$ & $\begin{array}{c}14.6072 \\
(0.0093) \\
{[5015]}\end{array}$ \\
\hline 1 & $\begin{array}{c}13.2416 \\
(0.025) \\
{[2.5]}\end{array}$ & $\begin{array}{c}13.8618 \\
(0.032) \\
{[3.5]} \\
\end{array}$ & $\begin{array}{c}14.1860 \\
(0.028) \\
{[5.5]}\end{array}$ & $\begin{array}{c}14.4244 \\
(0.033) \\
{[9.4]}\end{array}$ & $\begin{array}{c}14.4011 \\
(0.030) \\
{[17.1]} \\
\end{array}$ & $\begin{array}{c}14.5600 \\
(0.030) \\
{[53.3]}\end{array}$ \\
\hline 2 & $\begin{array}{c}13.2663 \\
(0.014) \\
{[4.0]}\end{array}$ & $\begin{array}{c}13.7919 \\
(0.017) \\
{[5.0]}\end{array}$ & $\begin{array}{c}14.1576 \\
(0.018) \\
{[7.0]}\end{array}$ & $\begin{array}{c}14.3781 \\
(0.020) \\
{[10.9]}\end{array}$ & $\begin{array}{c}14.5186 \\
(0.023) \\
{[18.5]}\end{array}$ & $\begin{array}{c}14.5955 \\
(0.020) \\
{[54.9]}\end{array}$ \\
\hline 4 & $\begin{array}{c}13.2590 \\
(-) \\
{[13.2]}\end{array}$ & $\begin{array}{c}13.8087 \\
(0.011) \\
{[14.3]}\end{array}$ & $\begin{array}{c}14.1568 \\
(0.013) \\
{[16.1]}\end{array}$ & $\begin{array}{c}14.3885 \\
(0.012) \\
{[20.1]}\end{array}$ & $\begin{array}{c}14.4937 \\
(0.012) \\
{[27.8]}\end{array}$ & $\begin{array}{c}14.5973 \\
(0.013) \\
{[63.7]}\end{array}$ \\
\hline 8 & - & $\begin{array}{c}13.8129 \\
(-) \\
{[34.4]}\end{array}$ & $\begin{array}{c}14.1729 \\
(0.0073) \\
{[35.9]}\end{array}$ & $\begin{array}{c}14.3947 \\
(0.0066) \\
{[39.6]}\end{array}$ & $\begin{array}{c}14.5176 \\
(0.0075) \\
{[47.3]}\end{array}$ & $\begin{array}{c}14.6003 \\
(0.0094) \\
{[83.6]}\end{array}$ \\
\hline 16 & - & - & $\begin{array}{c}14.1657 \\
(-) \\
{[77.9]}\end{array}$ & $\begin{array}{c}14.3854 \\
(0.0042) \\
{[80.3]} \\
\end{array}$ & $\begin{array}{c}14.5051 \\
(0.0053) \\
{[88.3]}\end{array}$ & $\begin{array}{c}14.5955 \\
(0.0052) \\
{[124.8]}\end{array}$ \\
\hline $\begin{array}{l}\text { Bench- } \\
\text { mark }\end{array}$ & $\begin{array}{c}13.2527 \\
(-) \\
{[1330]}\end{array}$ & $\begin{array}{c}13.7994 \\
(-) \\
{[3375]}\end{array}$ & $\begin{array}{c}14.1602 \\
(-) \\
{[7650]}\end{array}$ & - & - & - \\
\hline
\end{tabular}

Table 7: Lookback Call Options: Comparison of Plain and Bridge Monte Carlo

\begin{tabular}{|c|c|c|c|c|c|c|}
\hline \multicolumn{7}{|c|}{ Lookback call options: Efficiency gains. } \\
\hline K & 4 resets & 8 resets & 16 resets & 32 resets & 64 resets & 256 resets \\
\hline 1 & 5.3 & 4.4 & 6.9 & 5.5 & 7.2 & 9.0 \\
\hline 3 & 11 & 11 & 13 & 13 & 11 & 20 \\
\hline 4 & - & 9.2 & 11 & 20 & 28 & 40 \\
\hline 8 & - & - & 16 & 33 & 42 & 59 \\
\hline 16 & - & - & - & 40 & 45 & 129 \\
\hline
\end{tabular}

Table 8: Lookback Call Options: Efficiency Gains for Bridge Monte Carlo over Plain Monte Carlo 


\begin{tabular}{|c|c|c|c|c|c|c|}
\hline \multicolumn{6}{|c|}{ Up-and-In barrier call options: Times and standard deviations. } \\
\hline K & 4 resets & 8 resets & 16 resets & 32 resets & $\mathbf{6 4}$ resets & 256 resets \\
\hline \multirow{3}{*}{0} & 7.0347 & 7.2316 & 7.3727 & 7.4765 & 7.5351 & 7.5851 \\
& $(0.011)$ & $(0.011)$ & $(0.011)$ & $(0.011)$ & $(0.011)$ & $(0.011)$ \\
& {$[85.2]$} & {$[169.0]$} & {$[315.4]$} & {$[597.5]$} & {$[1157]$} & {$[4507]$} \\
\hline \multirow{3}{*}{1} & 7.0094 & 7.2556 & 7.4214 & 7.4392 & 7.5155 & 7.5433 \\
& $(0.018)$ & $(0.024)$ & $(0.028)$ & $(0.028)$ & $(0.027)$ & $(0.027)$ \\
& {$[2.1]$} & {$[2.8]$} & {$[\mathbf{4} .1]$} & {$[6.6]$} & {$[11.3]$} & {$[32.5]$} \\
\hline \multirow{3}{*}{2} & 7.0283 & 7.2211 & 7.4261 & 7.4640 & 7.5607 & 7.5608 \\
& $(0.014)$ & $(0.019)$ & $(0.023)$ & $(0.026)$ & $(0.024)$ & $(0.025)$ \\
& {$[3.6]$} & {$[4.4]$} & {$[5.7]$} & {$[8.1]$} & {$[12.8]$} & {$[34.0]$} \\
\hline \multirow{4}{*}{4} & 7.0261 & 7.2220 & 7.4015 & 7.4826 & 7.5713 & 7.6147 \\
& $(-)$ & $(0.014)$ & $(0.017)$ & $(0.021)$ & $(0.018)$ & $(0.017)$ \\
& {$[11.8]$} & {$[12.6]$} & {$[13.9]$} & {$[16.3]$} & {$[21.0]$} & {$[42.1]$} \\
\hline \multirow{4}{*}{8} & - & 7.2255 & 7.4021 & 7.5134 & 7.5316 & 7.6206 \\
& & $(-)$ & $(0.013)$ & $(0.018)$ & $(0.014)$ & $(0.015)$ \\
& & {$[28.0]$} & {$[29.3]$} & {$[31.8]$} & {$[36.4]$} & {$[57.8]$} \\
\hline \multirow{2}{*}{16} & - & - & 7.3772 & 7.4905 & 7.5456 & 7.5941 \\
& & & $(-)$ & $(0.008)$ & $(0.011)$ & $(0.013)$ \\
& & & {$[58.7]$} & {$[61.1]$} & {$[65.8]$} & {$[87.0]$} \\
\hline \multirow{2}{*}{ Bench- } & 7.0268 & 7.2348 & 7.3857 & & & \\
mark & $(-)$ & $(-)$ & $(-)$ & - & - & - \\
& {$[1180]$} & {$[2801]$} & {$[5860]$} & & & \\
\hline
\end{tabular}

Table 9: Up-and-In Barrier Call Options: Comparison of Plain and Bridge Monte Carlo. $\mathrm{B}=120$

\begin{tabular}{|c|c|c|c|c|c|c|}
\hline \multicolumn{7}{|c|}{ Up-and-In barrier call options: Efficiency gains. } \\
\hline K & 4 resets & 8 resets & 16 resets & 32 resets & 64 resets & 256 resets \\
\hline 1 & 15.2 & 12.7 & 11.9 & 14.0 & 17.0 & 23.0 \\
\hline 2 & 14.6 & 12.9 & 12.7 & 13.2 & 19.0 & 25.7 \\
\hline 4 & - & 8.3 & 9.5 & 10.1 & 20.6 & 44.8 \\
\hline 8 & - & - & 7.7 & 7.0 & 19.6 & 41.9 \\
\hline 16 & - & - & - & 18.5 & 17.6 & 37.1 \\
\hline
\end{tabular}

Table 10: Up-and-In Barrier Call Options: Efficiency Gains for Bridge Monte Carlo over Plain Monte Carlo 


\section{List of other working papers:}

\section{2}

1. Paolo Zaffaroni, Gaussian inference on Certain Long-Range Dependent Volatility Models, WP02-12

2. Paolo Zaffaroni, Aggregation and Memory of Models of Changing Volatility, WP02-11

3. Jerry Coakley, Ana-Maria Fuertes and Andrew Wood, Reinterpreting the Real Exchange Rate - Yield Diffential Nexus, WP02-10

4. Gordon Gemmill and Dylan Thomas, Noise Training, Costly Arbitrage and Asset Prices: evidence from closed-end funds, WP02-09

5. Gordon Gemmill, Testing Merton's Model for Credit Spreads on Zero-Coupon Bonds, WP0208

6. George Christodoulakis and Steve Satchell, On th Evolution of Global Style Factors in the MSCI Universe of Assets, WP02-07

7. George Christodoulakis, Sharp Style Analysis in the MSCI Sector Portfolios: A Monte Caro Integration Approach, WP02-06

8. George Christodoulakis, Generating Composite Volatility Forecasts with Random Factor Betas, WP02-05

9. Claudia Riveiro and Nick Webber, Valuing Path Dependent Options in the Variance-Gamma Model by Monte Carlo with a Gamma Bridge, WP02-04

10. Christian Pedersen and Soosung Hwang, On Empirical Risk Measurement with Asymmetric Returns Data, WP02-03

11. Roy Batchelor and Ismail Orgakcioglu, Event-related GARCH: the impact of stock dividends in Turkey, WP02-02

12. George Albanis and Roy Batchelor, Combining Heterogeneous Classifiers for Stock Selection, WP02-01

\section{1}

1. Soosung Hwang and Steve Satchell, GARCH Model with Cross-sectional Volatility; GARCHX Models, WP01-16

2. Soosung Hwang and Steve Satchell, Tracking Error: Ex-Ante versus Ex-Post Measures, WP01-15

3. Soosung Hwang and Steve Satchell, The Asset Allocation Decision in a Loss Aversion World, WP01-14

4. Soosung Hwang and Mark Salmon, An Analysis of Performance Measures Using Copulae, WP01-13

5. Soosung Hwang and Mark Salmon, A New Measure of Herding and Empirical Evidence, WP01-12

6. Richard Lewin and Steve Satchell, The Derivation of New Model of Equity Duration, WP0111

7. Massimiliano Marcellino and Mark Salmon, Robust Decision Theory and the Lucas Critique, WP01-10

8. Jerry Coakley, Ana-Maria Fuertes and Maria-Teresa Perez, Numerical Issues in Threshold Autoregressive Modelling of Time Series, WP01-09

9. Jerry Coakley, Ana-Maria Fuertes and Ron Smith, Small Sample Properties of Panel Timeseries Estimators with I(1) Errors, WP01-08

10. Jerry Coakley and Ana-Maria Fuertes, The Felsdtein-Horioka Puzzle is Not as Bad as You Think, WP01-07

11. Jerry Coakley and Ana-Maria Fuertes, Rethinking the Forward Premium Puzzle in a Nonlinear Framework, WP01-06

12. George Christodoulakis, Co-Volatility and Correlation Clustering: A Multivariate Correlated ARCH Framework, WP01-05 
13. Frank Critchley, Paul Marriott and Mark Salmon, On Preferred Point Geometry in Statistics, WP01-04

14. Eric Bouyé and Nicolas Gaussel and Mark Salmon, Investigating Dynamic Dependence Using Copulae, WP01-03

15. Eric Bouyé, Multivariate Extremes at Work for Portfolio Risk Measurement, WP01-02

16. Erick Bouyé, Vado Durrleman, Ashkan Nikeghbali, Gael Riboulet and Thierry Roncalli, Copulas: an Open Field for Risk Management, WP01-01

\section{0}

1. Soosung Hwang and Steve Satchell, Valuing Information Using Utility Functions, WP00-06

2. Soosung Hwang, Properties of Cross-sectional Volatility, WP00-05

3. Soosung Hwang and Steve Satchell, Calculating the Miss-specification in Beta from Using a Proxy for the Market Portfolio, WP00-04

4. Laun Middleton and Stephen Satchell, Deriving the APT when the Number of Factors is Unknown, WP00-03

5. George A. Christodoulakis and Steve Satchell, Evolving Systems of Financial Returns: AutoRegressive Conditional Beta, WP00-02

6. Christian S. Pedersen and Stephen Satchell, Evaluating the Performance of Nearest Neighbour Algorithms when Forecasting US Industry Returns, WP00-01

\section{9}

1. Yin-Wong Cheung, Menzie Chinn and Ian Marsh, How do UK-Based Foreign Exchange Dealers Think Their Market Operates?, WP99-21

2. Soosung Hwang, John Knight and Stephen Satchell, Forecasting Volatility using LINEX Loss Functions, WP99-20

3. Soosung Hwang and Steve Satchell, Improved Testing for the Efficiency of Asset Pricing Theories in Linear Factor Models, WP99-19

4. Soosung Hwang and Stephen Satchell, The Disappearance of Style in the US Equity Market, WP99-18

5. Soosung Hwang and Stephen Satchell, Modelling Emerging Market Risk Premia Using Higher Moments, WP99-17

6. Soosung Hwang and Stephen Satchell, Market Risk and the Concept of Fundamental Volatility: Measuring Volatility Across Asset and Derivative Markets and Testing for the Impact of Derivatives Markets on Financial Markets, WP99-16

7. Soosung Hwang, The Effects of Systematic Sampling and Temporal Aggregation on Discrete Time Long Memory Processes and their Finite Sample Properties, WP99-15

8. Ronald MacDonald and Ian Marsh, Currency Spillovers and Tri-Polarity: a Simultaneous Model of the US Dollar, German Mark and Japanese Yen, WP99-14

9. Robert Hillman, Forecasting Inflation with a Non-linear Output Gap Model, WP99-13

10. Robert Hillman and Mark Salmon, From Market Micro-structure to Macro Fundamentals: is there Predictability in the Dollar-Deutsche Mark Exchange Rate?, WP99-12

11. Renzo Avesani, Giampiero Gallo and Mark Salmon, On the Evolution of Credibility and Flexible Exchange Rate Target Zones, WP99-11

12. Paul Marriott and Mark Salmon, An Introduction to Differential Geometry in Econometrics, WP99-10

13. Mark Dixon, Anthony Ledford and Paul Marriott, Finite Sample Inference for Extreme Value Distributions, WP99-09

14. Ian Marsh and David Power, A Panel-Based Investigation into the Relationship Between Stock Prices and Dividends, WP99-08

15. Ian Marsh, An Analysis of the Performance of European Foreign Exchange Forecasters, WP99-07

16. Frank Critchley, Paul Marriott and Mark Salmon, An Elementary Account of Amari's Expected Geometry, WP99-06

17. Demos Tambakis and Anne-Sophie Van Royen, Bootstrap Predictability of Daily Exchange Rates in ARMA Models, WP99-05

18. Christopher Neely and Paul Weller, Technical Analysis and Central Bank Intervention, WP9904

19. Christopher Neely and Paul Weller, Predictability in International Asset Returns: A Reexamination, WP99-03 
20. Christopher Neely and Paul Weller, Intraday Technical Trading in the Foreign Exchange Market, WP99-02

21. Anthony Hall, Soosung Hwang and Stephen Satchell, Using Bayesian Variable Selection Methods to Choose Style Factors in Global Stock Return Models, WP99-01

\section{8}

1. Soosung Hwang and Stephen Satchell, Implied Volatility Forecasting: A Compaison of Different Procedures Including Fractionally Integrated Models with Applications to UK Equity Options, WP98-05

2. Roy Batchelor and David Peel, Rationality Testing under Asymmetric Loss, WP98-04

3. Roy Batchelor, Forecasting T-Bill Yields: Accuracy versus Profitability, WP98-03

4. Adam Kurpiel and Thierry Roncalli, Option Hedging with Stochastic Volatility, WP98-02

5. Adam Kurpiel and Thierry Roncalli, Hopscotch Methods for Two State Financial Models, WP98-01 